\title{
Detection of Submillimeter Polarization in the Orion Nebula
}

R.H. Hildebrand, M. Dragovan, and G. Novak

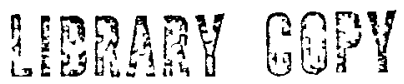

$\therefore 1024$

IANGLEY RESEARCH SENTER LIGRARY, NASA HAA"PTCN, VIRGINIA

\section{October 1984}

Natıonal Aeronautics and Space Adminıstratıon

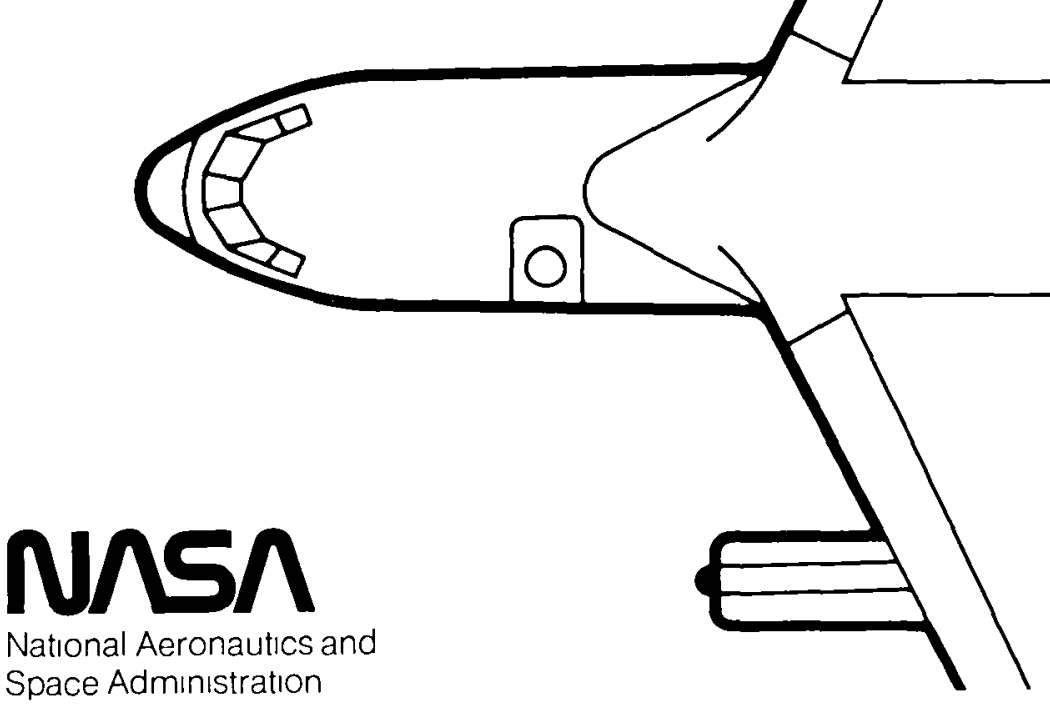




\title{
Detection of Submillimeter Polarization in the Orion Nebula
}

\author{
R. H Hildebrand \\ $M$ Dragovan \\ G. Novak, Unıversity of Chıcago, Chıcago, Illınıos
}


DETECTION OF SUBMILLIMETER POLARIZATION IN THE ORION NEBULA

R. H. Hildebrand, ${ }^{*} M$ Dragovan, and G. Novak

Department of Physics and

Enrico Ferml Inst1tute

The University of Chicago 


\section{ABSTRACT}

We have observed Iinear polarization of the submilifmeter $(270 \mu \mathrm{m})$ continuum radiation from two regions of Orfon: one centered on the Kleinmann-Low Nebula and one centered on the $400 \mu \mathrm{m}$ peak 1:5 south of the nebula. The polarizations measured for these regions are $P=(1.7 \pm 0.4) \%$ at $\phi=23^{\circ} \pm 7^{\circ}$ and $P=(1.7 \pm 0.5) \%$ at $\phi=27^{\circ} \pm 7^{\circ}$ respectively. We find a $2 \sigma$ upper limit, $P S 1.6 \%$, for the nebula $W 3(\mathrm{OH})$. The position angle at $K L$ is orthogonal to that measured at $11 \mu \mathrm{m}$ by Dyck and Befchman and at 11 and $20 \mu \mathrm{m}$ by Knacke and Capps. The far-IR values for $K L$ reported by Gull et al. $(\lesssim 2 \%)$ and by Cudlip et al. ("1-2\% level") are consistent with the submillimeter results. 


\section{INTRODUCTION}

It has long been recognized that interstellar clouds are potentlal sources of polarized emission at far-1nfrared and submillimeter wavelengths (Stein 1966). Dall'oglio et al. (1974) have discussed the polarization to be expected for thermal and non-thermal radiation at these wavelengths and have reported polarized signals of undetermined magnitude in several $1^{\circ}$ diameter fields of view for a wavelength band extending from 100 to $2000 \mu \mathrm{m}$.

The first measurements of the far-infrared polarization of an interstellar cloud were those obtained in the alrborne observations of Dennison et al. (1977) and Gull et al. $(1978,1980)$, who set an upper 11mit of $\sim 2 \%$ linear polarization for the Kleinmann-Low (KL) region in Orion for three wavelengths bands from 30 to $115 \mu \mathrm{m}$. The first positive result in the far infrared (at $\bar{\lambda}=80 \mu \mathrm{m}$ ) was obtalned in a balloon-borne observation, again for $\mathrm{KL}$, by Cudlip et al. (1982) who said: "Although the instrumental polarization could not be determined accurately during flight we can say with reasonable certainty that $M 42$ possesses far infrared polarization at the 1-2 per cent level." Assuming instrumental polarization as measured on the ground, they obtained a polarization of $(2.2 \pm 0.4) \%$ at a position angle, $16^{\circ} \pm 8^{\circ}$.

At submillimeter (SMM) wavelengths, the polarization should not necessarily be the same as in the far infrared. At the longer wavelengths, the increase in cloud transparency and in the relative efficlency for detection of cool radiators could change considerably the welghting given to different regions in a cloud, and, within a single region, the weighting to grain species of differing emissivities (e.g., Stein 1966; Mezger, Mthis, and Panagia 1982; Hildebrand 1983).

In this letter we present the first submillimeter polarization measurements: an upper linit for the nebula $W 3(\mathrm{OH})$ and clearly measurable polarization for two 90" diameter regions in Orion; one centered on the Kleinmann-Low Nebula (KL) and 
one centered on the cooler peak 1:5 south of $\mathrm{KI}$ appearing in the $400 \mu \mathrm{m}$ continuum map of Keene, Hildebrand, and Whitcomb (1982) and also in the molecular line maps of Thaddeus et al. $\left(1971 ; \mathrm{H}_{2} \mathrm{CO}\right)$, Clark, Buh1, and Snyder (1974; $\mathrm{HCN}$ ) and Zfurys et al. $\left(1981 ; \mathrm{NH}_{3}(2,2]\right)$.

\section{OBSERVATIONS AND RESULTS}

The observations were made in September 1983 and January 1984 with the NASA Kulper Airborne Observatory (KAO).

Different instruments, $M$ and $M$, were used in the September and January flights. The instruments were similar in effective wavelength (286 and $270 \mu \mathrm{m}$ respectively), in beam size (90"), in the use of ${ }^{3} \mathrm{He}$-cooled detectors, in providing for simultaneous measurement of two components of polarization, and in exposing the detectors only to $100 \%$ polarized radiation (Hildebrand 1984), but they differed considerably in other respects. In $M 1$, the plane of polarization was rotated by means of a sapphire half-wave plate; in $M$, by means of an Abbe/König K-mirror (e.g., Naumann 1967), a device which, unlike the half-wave plate, has the property of rotating the Image at twice the rate of the mechanical rotation. Other differences are described in a footnote to Table 1 . In September, the instrumental polarization was removed by subtraction of signals from Venus which were assumed to be unpolarized. Because of that uncertain assumption we have not combined the September results with those for January, which relied on rotation of the sky with respect to the instrument, a rotation easily achieved with the alt-azimuth telescope of the KAO. Despite these differences in instrumentation and technique the results agree within the errors. The $M$ polarimeter is shown schematically in Figure 1. ( $M$ is similar except as otherwise specified.) The $\mathrm{K}$-mirror was stepped in $15^{\circ}$ intervals, giving intervals of $30^{\circ}$ in the plane of polarization. The horizontal (H) and 
vertical ( $V$ ) components of the radiation transmitted by the $\mathrm{K}$-mirror were separated by a fixed parallel-wlre grid (Hertz 1889; Auton 1967) inclined at $45^{\circ}$ to the optic axis. The grid transmitted the $\mathrm{H}$ component, perpendicular to the grid wires, and reflected the $\mathrm{V}$ component, parallel to the wires. The two components were measured simultaneously.

The signals, $H$ and $V$, corresponding to these components were combined to form the "polarization signal" defined by $S(\theta, \alpha)=[(\mathrm{V}-\mathrm{H}) /(\mathrm{V}+\mathrm{H})]_{\theta, \alpha}$ where $\theta$ 1s the angle of rotation of the $K$-mirror and $\alpha$ is the known angle of the sky (north) with respect to the instrument (up). To insure an accurately known relationship between $\theta$ and $\alpha$, we made pre-flight measurements of $S(\theta, \alpha)$ with a $100 \%$ polarized source mounted directly in front of the secondary mirror of the telescope.

The polarization signal is nearly independent of changes in atmospheric absorption or emission, but does not directly give a true measure of the source polarization since it includes Instrumental effects produced by the telescope and by the polarimeter 1tself. The instrumental effects measured in flight were significantly different from those measured in the laboratory. In the January flight, we removed these effects (to first order) by taking the difference, $D\left(\theta, \alpha_{1}, \alpha_{2}\right) \equiv S\left(\theta, \alpha_{2}\right)-s\left(\theta, a_{1}\right)$, between values of $s$ measured in portions of the flight with values $\alpha_{1}$ and $\alpha_{2}$ of the sky rotation angle. The measured values of $D$ and the sine curves, or "difference curves," giving the best fits to those values are shown in Figure 2. Where $\alpha_{1}=\alpha_{2}$, the deviations of $D$ from a constant give the measurement noise (Figure $2 a$ ) or provide tests for instrumental effects (e.g. a change in chopping direction; Fig. 2b: The chopping secondary of the telescope switched at $27 \mathrm{~Hz}$ between the source position and two off-source positions displaced 300" in approximately the east and west directions). The polarization measurements $\left(\alpha_{1} \neq \alpha_{2}\right)$ for $\mathrm{KI}$ and the 400 m peak are shown in 
Figures $2 c$ and $2 d$. The degrees, $P$, and position angles, $\phi$, of the polarization derived from these measurements are presented in Table 1.

The first four lines of the table show the uncorrected results with statistical errors. The next six lines show a serles of tests for systematic effects based on changes in conditions between successive measurements of $s(\theta, \alpha)$. The tests included a $46^{\circ}$ change in chopping direction (line 5) and deliberate misalignments between measurements (11nes 6-8) by an amount (20") large compared to typical guiding errors $\left(\lesssim 5^{\prime \prime}\right)$ but enough smaller than a beam diameter (90") so that one should expect little real change in the polarization. A less severe test was to change sources: since the $\mathrm{KL}$ and $400 \mu \mathrm{m}$ peaks gave nearly the same results individually, the values computed by subtracting the first measurement of one from the second measurement of the other tested the effect of the differences in the intensity distributions around the two peaks (1ines 9,10). On the basis of these tests, we estimate systematic errors of $0.4 \%$ in $P$ and $7^{\circ}$ in $\phi$ under the conditions on the measurements. The last three lines of the table give our best estimates for the January ' 84 results, taking 1nto account all sources of error and a small correction due to incomplete removal of instrumental polarization in the difference curve.

A laboratory test has demonstrated that the technique used in January gives results in agreement with those obtained by an independent method. A sheet of fluorogold, a common submillimeter filter material which is slightly polarizing, was placed in front of a blackbody source to simulate a polarized cloud. The direction and magnitude of the polarization of the fluorogold sheet were first measured by rotating the sheet while keeping a parallel wire grid fixed in front of the polarimeter and keeping the K-mirror fixed; i.e., the polarimeter was used only as a photometer recelving $100 \%$ polarized radiation in a fixed direction. The wire grid was then removed and with the fluorogold first at $\alpha_{1}=0^{\circ}$ and then 
at $\alpha_{2}=45^{\circ}$ to simulate rotation of the sky between two legs of a flight, the $\mathrm{K}$-mirror was stepped in $\theta$ and the polarization was computed from the difference curve as in the flights. The direction and degrees of polarization measured by the two methods were in good agreement $\left[(6.0 \pm 0.3) \%\right.$ at $0^{\circ} \pm 5^{\circ}$ and $(6.03 \pm 0.13) \%$ at $-4.1^{\circ} \pm 0.6^{\circ}$ for the first and second methods, respectively].

\section{DISCUSSION}

The inftial goal of this investigation has been to see whether there exists a measurable effect at submillimeter wavelengths.

Since we do find a measurable effect, it should be feasible to map the polarization and thereby infer the configuration of magnetic fields in clouds. If submillimeter polarization is due to emission from spinning grains where damping processes have removed components of the spin perpendicular to the principal axis of greatest inertia and perpendicular to the magnetic field (Purcell 1979, Davis and Greenstein 1951), then the position angle of the submillimeter polarization should be perpendicular to the transverse component of the field. It should be feasible to investigate whether the field direction is correlated with the rotational axis, outflow lobes, or other features of a dense cloud. The submillimeter results should be free of contributions from scattering or selective absorption and should sample the entire depth of a cloud.

Submillimeter polarimetry may also be used to study grain alignment mechanisms. Polarimetry of suitably chosen sources should test whether differences in gas and dust temperature, differences in the bulk motion of gas and dust, or the presence of atomic hydrogen (for conversion to molecules at preferred grain sites) are necessary conditions for alignment, and the extent to which the growth of grain mantles Interferes with alignment (Aannestad and 
Greenberg 1983; Gold 1952; Johnson 1982; Johnson et al. 1981; Purcell 1979; Spitzer and MGlynn 1979).

In addition, polarimetry at submillimeter and far-IR wavelengths may be used to study grain properties. In reglons remote from heating sources, individual dust grains may be at considerably different temperatures due to differences in emissivity. Measurements on the high (low) frequency side of the thermal emission peak will favor detection of radiation from low (high) emissivity grains. Polarimetry at different wavelengths may therefore show correlations between grain properties related to temperature (dielectric constant and size) and those related to grain alignment and emission of polarized radiation (magnetic susceptibility and shape).

The Orion/KL results of Knacke and Capps (1979) at 11 and $20 \mu \mathrm{m}$ show polarization ranging from $\sim 7 \%$ near the Becklin Neugebauer Object (BN) to $\sim 2 \%$ at a point 16" south of $B N$. The mean values for the five measured points, computed by adding Stokes parameters, are $P=4 \%, \phi=103^{\circ}$ at $11 \mu \mathrm{m}$ and $\mathrm{P}=4 \%, \phi=118^{\circ}$ at $20 \mu \mathrm{m}$. These measurements are within the silicate resonances and hence favor detection of polarization due to selective absorption by aligned silicate grains on the near side of the nebula (Dyck and Belchman 1975; Dennison 1977).

The SMM measurements detect emitted radiation, they cover a much wider area (each beam diameter $=90^{\prime \prime} \approx 0.2$ parsec), they sample the entire depth of the cloud (probably $\gg .2$ parsec), and being far removed from the sllicate resonance features, they may include a greater fraction of radiation from non-silicate grains such as graphite or amorphous carbon, materials less susceptible to paramagnetic relaxation (graphite is very slightly diamagnetic). Considering these differences, it is perhaps surprising that the polarizations at SMM and IR wavelenths should be nicely orthogonal and of the same order of magnitude as would be expected if the polarization at 11 and $20 \mu \mathrm{m}$ 1s due to selective 
absorption through 1-2 optical depths by aligned grains and the SMM polarization 1s due to emission from the same grains.

We may infer from the SMM measurements that the magnetic field must have some degree of uniformity on a scale comparable to the dimensions of the two nearly adjacent SMM detector beams $(\sim 2 \times 0.2$ parsec $)$. We may also infer that the speclal conditions of shocked gas and large velocity dispersion observed at $\mathrm{KL}$ and presumably absent at the $400 \mu \mathrm{m}$ peak are not necessary for polarized SMM emission.

Erickson et al. (1982) have observed bipolar c0 outflow of high velocity in the kL region. The outflow lobes are much smaller $\left(\sim 30^{\prime \prime}\right)$ than the two 90" diameter regions observed with the SMM polarimeter. The axis of the outflow lobes is approximately along the direction $\phi \pm 90^{\circ}$ of the magnetic field given by the SMM measurements. This relationship is consistent with the assumption that the outflow is correlated with the pre-existing direction of the fleld (Königl 1982; Draine 1983).

\section{ACKNOWLEDGMENTS}

We are grateful to $S$. Lucero for the mechanical design of the polarimeter, to A. Stark for calling our attention to the K-mirror, to the staff of the Kuiper Airborne Osservatory for their support, to J. A. Davidson, D. A. Harper, D. T. Jaffe, and R. Loewenstein for assistance during the observations, to M. Harwit, D. T. Jaffe, and an anonymous referee for comments on a draft of this paper, and to A. Königl for helpful discussions. Th1s work was supported by NASA grant NSG 2057. 
REFERENCES

Aanestad, P. A., and Greenberg, J. M. 1983, Ap. J., 272, 551.

Auton, J. P. 1967, App1. Opt., 15, 53.

Clark, F. O., Buhl, D., and Snyder, L. E. 1974, Ap. J. 190, 545.

Cudlip, W., Furniss, I., King, K. J., and Jenings, R. E. 1982, M.N.R.A.S., 200, 1169.

Dall'oglio, G., Melchiori, B., Melchiori, F., Natale, V., Aello, S., and Mencaralia, F. 1974, in Planets, Stars, and Nebulae Studied with Photo polarlmetry, ed, T. Gehrels (Arizona).

Dav1s Jr., L. and Greenste1n, J. L., 1951 Ap. J., 114, 206.

Dennison, B. 1977, Ap. J., 215, 529.

Dragovan, M. 1984, Submitted to Applled Opt1cs.

Draine, B. J. 1983, Ap. J., 270, 519.

Dyck, H. M., and Beichman, C. A. 1974, Ap. J., 194, 57.

Erlckson, N. R., Goldsmith, P. F., Snell, R. L., Berson, R. L., Huguenin, G. R., Ulich, B. L., and Lada, C. J. 1982, Ap. J., 261, L103.

Gu11, G. E., Houck,J. R., McCarthy, J. F., Forrest, W. J., and Harwit, M. 1978, A. J., $83,1440$.

Gull, G. E., Russell, R. W., Melnick, G., and Harwit, M. 1980, A. J., 85, 1379. Hertz, H. R. 1889, Annalen der Physik, 36, 769.

Hildebrand, R. H. 1983, Q. Jl. R. astr. Soc., 24, 267. Hildebrand, R. H. 1984, in International Conference on Nonimaging Concentrators, ed. M. C. Ruda, Proc. SPIE, 441, 40. Johnson, P. E. 1982, Nature, 295, 371. 
Johnson, P. E., Rieke, G. H., Lebofsky, M. J., and Kemp, J. C. 1981, Ap. J., 245,871 .

Keene, J., Hildebrand, R. H., and Whitcomb, S. E. 1982, Ap. J. (Letters), 252, L11.

Knacke, R. F., and Capps, R. W. 1979, A. J., 84, 1705.

König1, A. 1982. Ap. J., 261, 115.

Mezger, P. G., Mathis, J. S., and Panagla, N. 1982, A.\& A., 105, 372.

Naumann, H. 1967, Splegelnde Prismen, in Handbuch der Physik, 29, ed. S. Flugge

(Berlin: Springer Verlag), p. 217.

Purcell, E. M. 1979, Ap. J., 231, 404.

Spitzer, L., and MeGlynn, T. A. 1979, Ap. J., 231, 417.

Stein, W. 1966 Ap. J., 144, 318.

Thaddeus, P., Wilson, R. W., Kutner, M., Penzlas, A. A., and Jefferts, K. B. 1971, Ap. J. (Letters), 168, L59.

Ziurys, L. M., Martin, R. N., Pauls, T. A., and Wilson, T. L. 1981 Astron. Astrophys., $104,288$. 
FIGURE LEGENDS

Figure 1. Schematic diagram of polarimeter $\mathbb{R}$. The incoming radiation passes through a spectral filter, F, and then through an Abbe/König $\mathrm{K}$-mirror, K. The K-mirror is rotated about the optic axis in $15^{\circ}$ steps giving $30^{\circ}$ steps in the plane of polarization of the transmitted radiation. A fixed parallel wire grid, $G_{1}$, separates the vertical, $\mathrm{V}$, and horizontal, $\mathrm{H}$, components. The light paths are folded (and additional short wavelength filtering is provided) by reflections from the fixed grids $G_{2}$ and $G_{3}$ which have wires parallel to the planes containing the $\mathrm{V}$ and $\mathrm{B}$ components respectively. The beams are concentrated using Winston concentrators and detected by ${ }^{3} \mathrm{He}$-cooled bolometers $\mathrm{B}_{\mathrm{V}}$ and $\mathrm{B}_{\mathrm{H}}$.

Figure 2. Curves showing the difference, $D\left(\theta, \alpha_{1}, \alpha_{2}\right)$, between the polarization signals $(V-H) /(V+H)$ at sky rotation angles $\alpha_{1}$ and $\alpha_{2}$ where $\theta$ is the angle of the $\mathrm{K}$-mirror. The source polarizations derived from the curves are shown in Table 1.

(a) Measurement noise $\left(\alpha_{1}=\alpha_{2}\right.$; no change in conditions).

(b) Test for the effect of a $46^{\circ}$ change in the chopping direction $\left(\alpha_{1}=\alpha_{2}\right)$

(c) Measurement of the polarization of $\mathrm{KL}\left(\alpha_{2}-\alpha_{1}=46^{\circ}\right)$.

(d) Measurement of the polarization at the $400 \mu \mathrm{m}$ peak $\left(\alpha_{2}-\alpha_{1}=41^{\circ}\right)$. 
POSTAL ADDRESS

MARK DRAGOVAN, ROGER H. HILDEBRAND, and GILES NOVAK: The Enrico Ferm1 Inst1tute, The University of Chicago, 5640 South Ellis Avenue, Chicago, IL 60637. 
TABLE I

$270 \mu \mathrm{m}$ POLARIMETRY OF ORION AND W3(OH)

\begin{tabular}{|c|c|c|c|c|c|c|c|}
\hline \multirow[t]{2}{*}{ Line } & \multicolumn{2}{|c|}{ Source ${ }^{a}$} & \multicolumn{2}{|c|}{ Polarization ${ }^{b}$} & \multirow[t]{2}{*}{ Date } & \multirow{2}{*}{$\begin{array}{l}\text { Instru- } \\
\text { ment }^{c}\end{array}$} & \multirow[t]{2}{*}{ Comments } \\
\hline & Leg 1 & Leg 2 & $P(\%)$ & $\phi$ & & & \\
\hline 1 & $\mathrm{KI}$ & - & $2.6 \pm 0.6$ & $16^{\circ} \pm 6^{\circ}$ & $\begin{array}{l}\text { Sept. } \\
1983\end{array}$ & MI & $\begin{array}{l}\text { Venus used as } \\
\text { calibrator. } \\
\text { Statistical errors. }\end{array}$ \\
\hline 2 & KL & $\mathrm{KL}$ & $1.8 \pm 0.2$ & $23^{\circ} \pm 3^{\circ}$ & $\begin{array}{l}\text { Jan. } \\
1984\end{array}$ & $M$ & No callbrator \\
\hline 3 & KHW & KHW & $1.8 \pm 0.3$ & $27^{\circ} \pm 5^{\circ}$ & & & assumed. \\
\hline 4 & W3 $(\mathrm{OH})$ & W3 (OH) & $<1.6(2 \sigma$ & $11 \mathrm{~m} 1 \mathrm{t})$ & & & Statistical errors. \\
\hline 5 & $\mathrm{KL}\left(120^{\circ}\right)$ & $\mathrm{KL}\left(166^{\circ}\right)$ & $1.9 \pm 0.2$ & $25^{\circ} \pm 4^{\circ}$ & $\begin{array}{l}\text { Jan } \\
1984\end{array}$ & M & Tests for systema- \\
\hline 6 & $K L(20 " W)$ & $\mathrm{KL}$ & $2.2 \pm 0.4$ & $25^{\circ} \pm 6^{\circ}$ & & & tic effects. \\
\hline 7 & $\mathrm{KL}(20 \cdot \mathrm{E})$ & $\mathrm{kL}$ & $1.3 \pm 0.4$ & $7^{\circ} \pm 9^{\circ}$ & & & Statistical errors. \\
\hline 8 & $\mathbf{K L}$ & $\mathrm{KL}\left(20^{\prime \prime S}\right)$ & $2.2 \pm 0.5$ & $32^{\circ} \pm 7^{\circ}$ & & & \\
\hline 9 & KHW & $\mathrm{KL}$ & $1.7 \pm 0.5$ & $20^{\circ} \pm 8^{\circ}$ & & & \\
\hline 10 & $k \mathbb{L}$ & KHW & $1.9 \pm 0.6$ & $29^{\circ} \pm 9^{\circ}$ & & & \\
\hline 11 & $\mathrm{~K} L$ & $\mathrm{KL}$ & $1.7 \pm 0.4$ & $23^{\circ} \pm 7^{\circ}$ & $\begin{array}{l}\text { Jan. } \\
1984\end{array}$ & M & Corrected results. \\
\hline 12 & KHW & KHW & $1.7 \pm 0.5$ & $27^{\circ} \pm 7^{\circ}$ & & & Total errors. \\
\hline 13 & $\mathrm{~W} 3(\mathrm{OH})$ & W3 $(\mathrm{OH})$ & $<1.6(2 \sigma$ & $\operatorname{limit})$ & & & \\
\hline
\end{tabular}

a"Leg 1" and "Leg 2" refer to segments of the flight at different rotations of the sky with respect to the instrument. Except for the Sept. ' 83 results, the polarization is derived from the difference of the data for the two legs. The chopping direction is the same for both legs except where angles are indicated (1ine 5). The source is centered except where displacements are indicated (11nes $6,7,8)$. KL Is the Kleinmannn-Low Nebula. KHW is the $400 \mu \mathrm{m}$ peak 1:5 south of $\mathrm{KL}$ (Keene, Hildebrand, and Whitcomb 1982). 


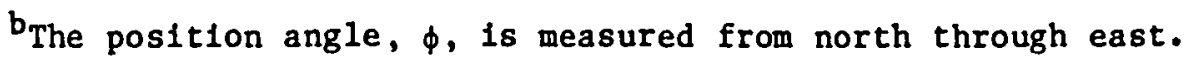

In M, the plane of polarization is rotated by means of a sapphire half wave plate; the passband, 263-308 $\mu \mathrm{m}$ fwhm $\left(\lambda_{\text {eff }}=286 \mu \mathrm{m}\right)$, is defined by a double half wave filter (Dragovan 1984); the light path is folded with front surface silver mirrors. In M2, the plane of polarization is rotated by a K-mirror (Naumann 1967); the passband, 200-400 $\mu \mathrm{m}$ fwhm $\left(\lambda_{\text {eff }}=270 \mu \mathrm{m}\right)$, is defined by capacitive grid filters and diffraction; the light path is folded with parallel-wire grids. 


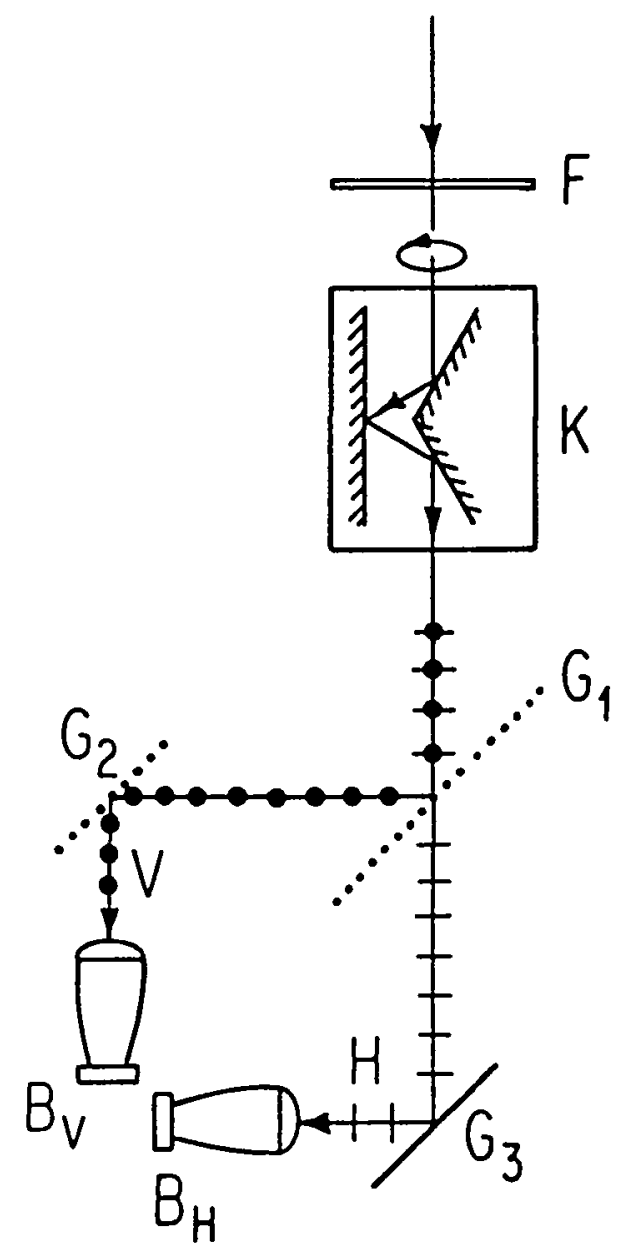

Fig. I 

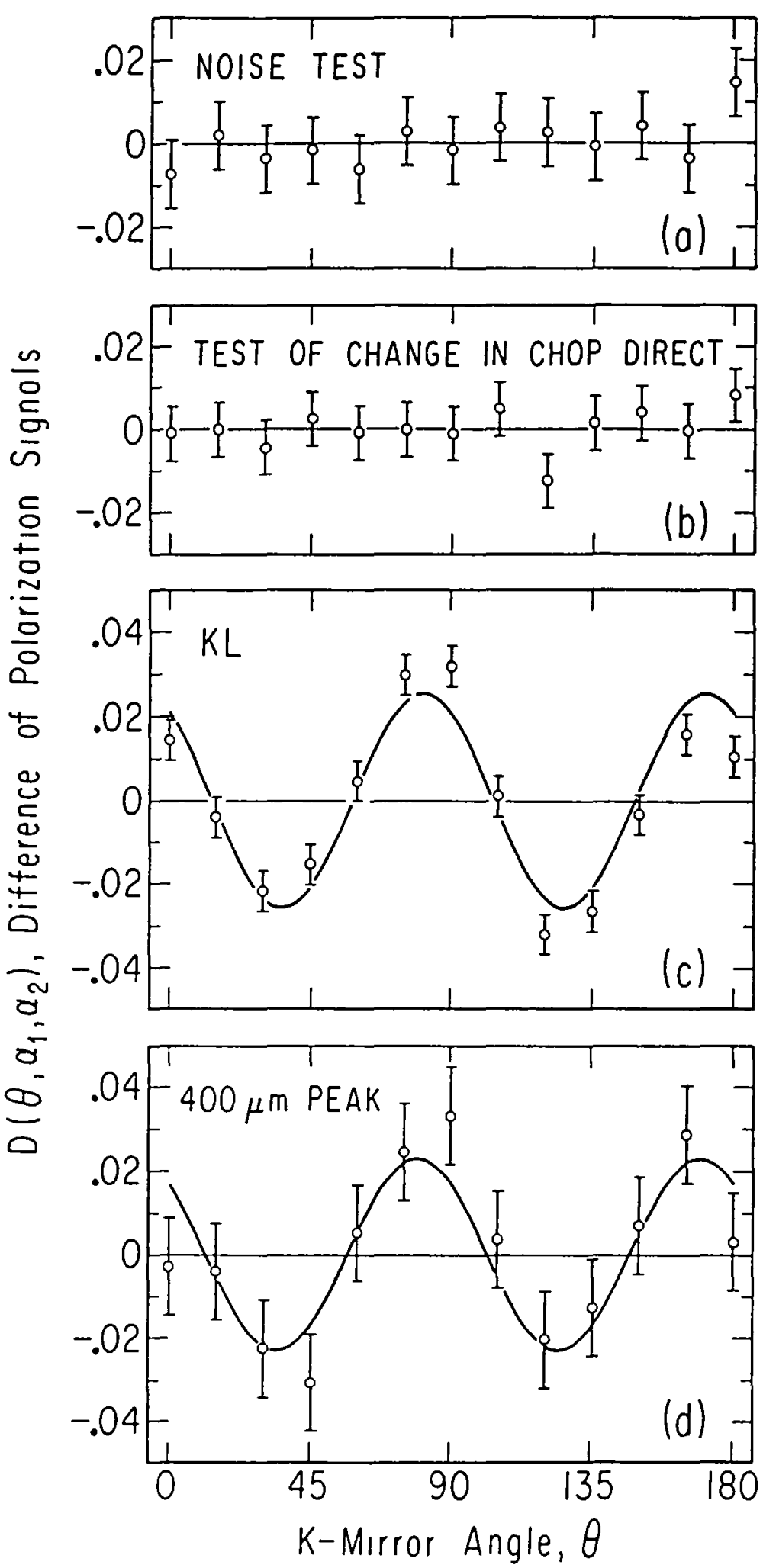

Fig.2 


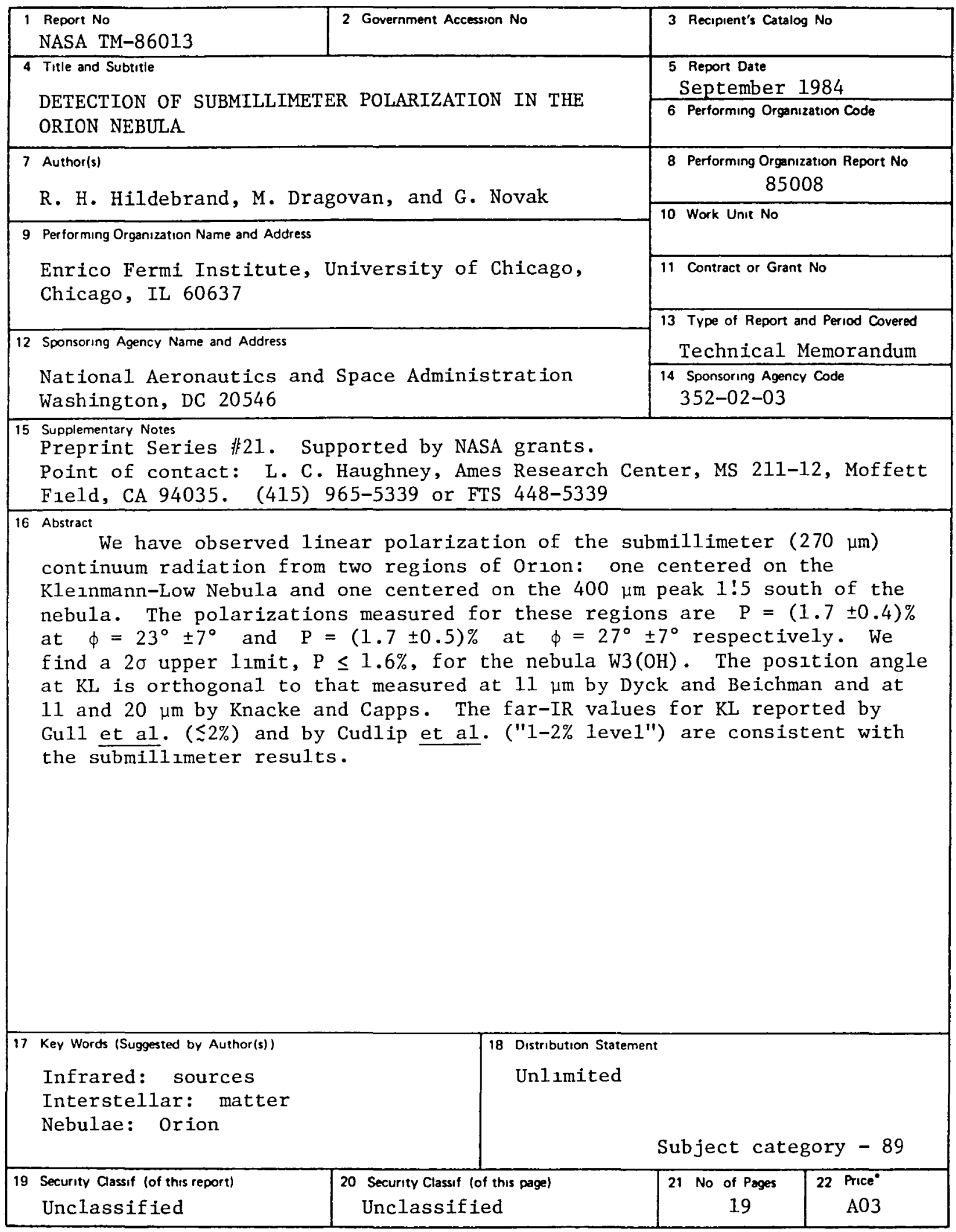

- For sale by the Natıonal Technical Information Service, Springfield, Virginia 22161 
End of Document 